\title{
Efficient biscale design of semiregular porous structures with desired deformation behavior
}

\author{
Chao $\mathrm{Xu}^{\mathrm{a}}$, Ming $\mathrm{Li}^{*}, \mathrm{a}$, Jin Huang ${ }^{\mathrm{a}}$, Shuming $\mathrm{Gao}^{\mathrm{a}}$ \\ ${ }^{a}$ State Key Laboratory of CADECG, Zhejiang University, Hangzhou, China
}

\begin{abstract}
Porous materials/structures are observed in many natural objects, exhibit exceptional properties, and have wide industrial applications. The rapid advance in additive manufacturing has also facilitated the process to fabricate such complex porous structures with fine accuracy control. However, the design of optimal porous structures with the desired deformation behavior is still very challenging because of its high computational complexity. In this study, a novel and efficient approach was developed to solve this problem by designing a semiregular porous structure with a hole within each mesh element of a given quad/hex-mesh layout of the design domain. The proposed approach ultimately analytically expresses the displacement of the design model as a function of the design variables, and consequently solves the optimization problem of porous structure design efficiently. The novelty was mainly achieved by representing a compact design space, building approximate analytical structure-property relation, and estimating the displacement variations of material property. The good property was achieved using the advanced numerical techniques: model reduction, homogenization theory, metamodeling, and the combined approximations. Various numerical examples were also tested to demonstrate the performance of the approach.
\end{abstract}

Key words: semiregular structure, porous structure, design optimization, model reduction, homogenization, additive manufacturing

\section{Introduction}

With the development of additive manufacturing technology, many research studies have been conducted to explore its potential utilities to product design and manufacturing. One of the most exciting problems is to design internal structures that achieve the desired physical properties. The main challenges are to (i) find an effective representation of the structure, (ii) accurately predict the relationship between the physical properties and structure, and (iii) design highly efficient numerical methods for structure optimization under specific constraints.

In this study, we show that the novel semiregular porous structure has great advantages of low-dimensional presentation, accurate property and performance prediction, and efficient optimization. Porous structures are observed in many natural materials or objects, such as human bones, woods, plant stems, and bird beaks. Porous structures are produced by million years natural evolution, exhibit exceptional properties [1,2] in the case of wave absorption $[3,4]$, shock resistance $[5,6]$, damping enhancement $[7,8]$, defect tolerance $[9,10,11]$, and multifunctional usage $[12,13,14,15,16]$, at a relatively low density. These superior properties are very important for industrial usage, and have attracted much research interest.

${ }^{*}$ Corresponding author: liming@cad.zju.edu.cn
According to the cell arrangements, two main categories of engineered porous structures have been studied previously: periodic and stochastic. Both of them have wide industrial applications. See the first two structures in Fig. 1. The periodically arranged regular structures were first studied, where completely identical cell elements (called Representative Volume Element(RVE)) are assumed to be periodically distributed under periodic physical boundary conditions. Based on this assumption, various analytical or numerical homogenization theories have been proposed to predict the associated material properties [17]. The design of a topological structure for such an RVE of extreme material properties, such as negative Poisson's ratio, has also been recently studied [18].

Among the excellent works in studying periodic structures, Bertoldi and co-workers showed the great potential of these structures both in 2D [19, 20, 21] and $3 \mathrm{D}$ [22] spaces. When exploring a nonlinear adaptive response $[19,20]$ of structures, they identified procedures to guide the design of $3 \mathrm{D}$ soft and active metamaterials, where buckling provides dramatic changes in the structures [21, 23, 24]. Bertoldi's work opened the way for performance-oriented cellular structure design and inspired more work on this issue afterwards. However, these studies mainly focused on the experimental studies of such cellular structures, and did not explore approaches to automatically design and optimize such

October 25, 2016 
structures.

Besides these regular porous structures, randomly arranged porous structures and their associated physical properties have also been studied, mainly in the computational material communities [25]. From the material composition knowledge, the relation between the physical property and the microstructure distribution was mainly studied statistically, i.e., predicting the macroproperty in terms of the statistical parameters of the microstructures [26]. Approaches have also been proposed to find such optimal statistical parameters for performance optimization [27].

Unlike the two main types of porous structures, the study focuses on a novel semiregular porous structure, as shown in Fig. 1(c). This has a similar but not identical cellular shapes arranged at a certain layout control. The semiregular structure does not only consider the geometry of cellular elements, but also their global arrangements. This is very different from the regular porous structures whose layouts have been fully determined, and also very different from the stochastic porous structures, which only consider the statistical information of the cells' arrangement. As further explored in this study, the semiregular structure also allows a possible efficient physical simulation owing to its special geometric structures. The differences among the three types of porous structures shown in Table 1 were compared.

Based on the above observations, the problem of designing semiregular porous structures for the desired deformation behaviors of linear elasticity was studied, specifically, certain vertices of the porous structure deforming to specified locations under given external forces. The design problem has wide applications in medical or engineering industrials [28]. Owing to the special structure representations of semiregularity, the problem was reformulated as a biscale design problem, by building sequentially the structure-property relationship, and the property-performance relationship. Ultimately, the displacement of the design model is expressed as an analytical function in terms of the design variables using advanced numerical techniques such as reduced structure representations, homogenization theory and metamodeling [29, 30, 31], and combined approximation (CA) [32]. Based on this design, the problem of semiregular porous structure can be efficiently solved as an optimization problem. Various numerical examples were also tested to demonstrate the performance and efficiency of the approach.

The remainder of the paper is arranged as follows: The related work is first given in Section 2. The design problem and overall approach are described in Section 3. Then, the technical details are described in detail in Sections 4 and 5. Various numerical examples are also shown in Section 6 to test the accuracy and efficiency of the approach. The conclusion is given in Section 7.

\section{Related studies}

Porous structure design has attracted much interest in different research communities such as computational physics, mechanical engineering, computational materials and computer graphics. The research work closely related to the problem includes topology optimization, descriptor-based material design optimization, and fabrication-oriented design optimization.

\subsection{Descriptor-based material design optimization}

The use of computational tools or informatics knowledge to accelerate the process of novel material discovery is a long-term goal in material research. The stochastic porous structures are mainly studied using statistical tools, probably because of the limitations of material fabrication technology at the beginning. Kalidindi and co-workers $[25,33,34]$ conducted a systematic study on microstructure-sensitive design for performance optimization. The main idea behind their work was to represent the distribution of cells or their relative locations in the spectral space, called first-order, or second-order descriptors, using a small number of descriptor parameters. Based on this, the structure-property relationship can be expressed using an explicit function based on homogenization theory. Chen and co-workers also systematically studied problem of stochastic multiscale material design [35, 36, 37].

These studies have provided promising results and shown practical engineering applications, but are still limited in several aspects. First, the statistical geometry representations lose the geometric detail information, and consequently the predicted physical property may vary in a large range and even has no practical application. Second, even if the statistical descriptors are successfully derived, reconstructing the microstructure from them is still challenging - usually not unique and expensive to compute, particularly for higher-order descriptors.

\subsection{Topology optimization}

Topology optimization aims to find the optimal solidvoid material distribution within a design domain for structure performance optimization. Various approaches have been proposed to solve this problem including the homogenization-based approach, SIMP, level set, ESO; see Ref. [38] for a recent review. Basically, the approaches iteratively update the design's geometry after simulating its physical behavior until convergence, and are computationally very expensive.

These topology optimization approaches were initially studied at the single macroscale [38]. Later on, approaches to design an optimal RVE were proposed, without considering the macrostructure. The concurrent topology optimization approaches were also proposed to simultaneously optimize the macroscale and microscale topology [39] and are much more expensive to compute. Zhang et al. proposed an approach to design the porous 


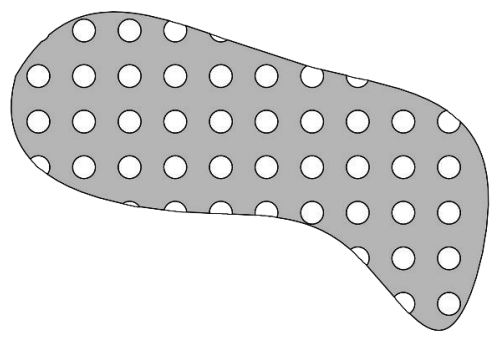

(a) Regular

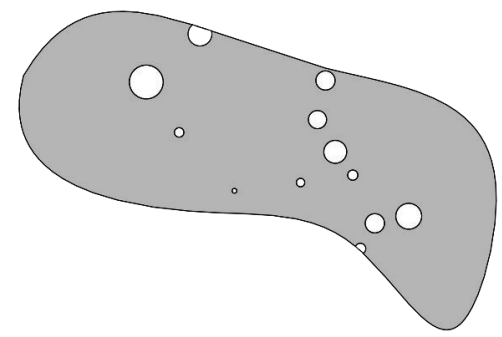

(b) Stochastic

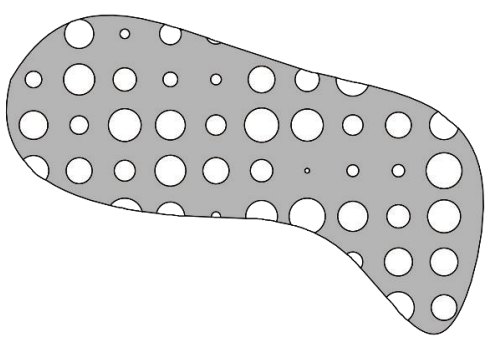

(c) Semiregular

Figure 1: Porous structures: regular, stochastic or semiregular structures.

Table 1: Difference between regular, stochastic and semiregular structures.

\begin{tabular}{|c|c|c|c|c|c|}
\hline Types & Geometry (1) & Arrangement (2) & Design DOFs & Representation & Physical property \\
\hline Regular (a) & Identical & Along X, Y, Z axis & Only (1) & Compact & Homo., exact \\
\hline Random (b) & Random & Random & $(1),(2)$ without exact control & Statistical & Homo., large range \\
\hline Semiregular (c) & Similar & Given & $(1),(2)$ with exact control & Reduced & Homo., approximate \\
\hline
\end{tabular}

structure of variable density for compliance minimization with a regular hex-mesh configuration and is very related to the work proposed here [40]. In this work [40], the microstructure and material properties were first built using the homogenization theory. Then, the conventional topology optimization approach was used to generate the cell density distribution, which was then mapped to cell structural parameters (circle/sphere radius here, for example). However, the previous work only studied the problem of compliance minimization, whereas the desired behavior is studied here. Moreover, owing to the introduction of various numerical techniques, the porous structure optimization is efficiently resolved.

\subsection{Performance-oriented structure optimization}

The design of the optimal topology of a structure, particularly for additive manufacturing, has also attracted much interest in geometric computing and computer graphics. Wang et al. optimized skin-frame structures with the interior of model via updating the radii and numbers of struts of an initial frame [41]. A honeycomblike Voronoi structures was introduced by Lu et al. [42] by adaptively updating the centroidal Voronoi diagram and its interior holes from an initially constructed Voronoi cells. These approaches initiated the studies on interior design in the graphics community, but do not fully address the optimization process, and the aspect of computational cost. Recently, Panetta et al. [43] and Schumacher et al. [44] also proposed excellent approaches for microstructure design for elastic performance control. They basically constructed a library of microstructures by topology optimization or geometric approaches, and then properly placed these structures with each prescribed hexahedron cell according to the design requirements or users' prescriptions. However, improper connection between the adjacent cells may appear, and the computations are also expensive.

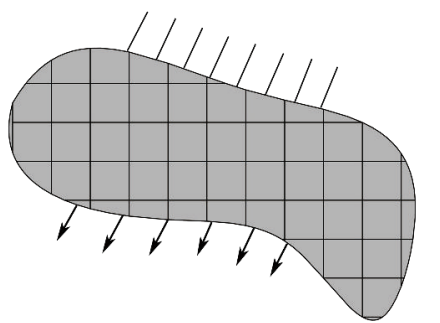

(a) Input: load, boundary conditions, material properties and grid

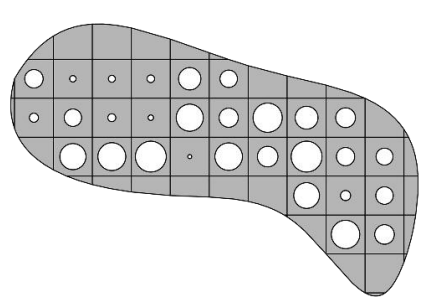

(b) Output: hole distribution
Figure 2: A simple illustration of the design problem input and output.

Model reduction is a commonly used technique in mechanical engineering or geometric computing [28, 45]. $\mathrm{Xu}$ et al. [28] demonstrated an interactive method to design the Young's modulus distribution of a material, and Li [45] proposed an approach for elastic animation editing with space-time constraints based on rotation-strain (RS) coordinates. In these approaches, model reduction was performed in the physical space, and the problem of porous structure construction was not studied.

\section{Problem statement and approach overview}

A linear elasticity problem was studied here: Design a semiregular porous structure so that the displacements of some of its points are close to certain values, under prescribed external forces. See also Fig. 2. In the following, the basics of the problem are first explained. Then, the problem of semiregular porous structure design is mathematically defined, followed by the approach overview.

\subsection{Semiregular structure and linear elasticity \\ 3.1.1. Semiregular structure}

A regular porous structure is a set of completely identical connected porous cells arranged under a specified order, 
as illustrated in Fig. 1(a).

A stochastic porous structure is a set of connected porous cells arranged randomly without any order, as illustrated in Fig. 1(b).

A semiregular porous structure is a set of connected porous cells of similar geometries arranged under a specified order, as illustrated in Fig. 1(c).

\subsubsection{Notations about the design domain}

$\Omega$ is the solid domain under study (as in Fig. 4(a));

$\Gamma_{0}$ is the fixed boundary of $\Omega$;

$\Gamma_{N}$ is the boundary of $\Omega$ exerted by external forces $\tau$;

$\Gamma_{\mathbf{D}}$ is the boundary of $\Omega$ when the displacements are set as the design target $\mathbf{u}_{\mathbf{D}}$;

$\Omega_{\mathcal{M}}$ is the quadrilateral mesh associated to $\Omega$ (as in Fig. 4(c));

$\mathbf{e}=\left\{e_{i}, 1 \leq i \leq n\right\}$ is the quad mesh set; $e_{i} \in \mathbf{e}$ for the $i$-th mesh element; $e \in \mathbf{e}$ for a general mesh element;

$\mathbf{r}=\left\{r_{\min } \leq r_{i} \leq r_{\max }, 1 \leq i \leq n\right\}$ is the radius distribution over model $\Omega_{\mathcal{M}}$;

$\Omega_{\mathcal{M}, \mathrm{r}}$ is the semiregular porous structure obtained by digging a hole within the $i$-th element $e_{i}$ of radius $r_{i} \in \mathbf{r}$ (as in Fig. 4(e));

$\Omega_{\mathcal{M}, \rho}$ is a density model of prescribed density distributions $\rho$ (as in Fig. 4(g));

$\left\{\boldsymbol{\rho}_{k}\right\}_{k=0}^{N}$ are the density bases after the model reduction, for the number of bases $N$.

\subsubsection{Linear elasticity}

Following the principle of linear elasticity studied here, the deformation of $\Omega$ can then be stated as follows: Find the displacement field $\mathbf{u}$ satisfying the following equations including a PDE (partial differentiable equation).

$$
\begin{cases}-\operatorname{div} \sigma(\mathbf{u})=\mathbf{f}, & \text { on } \Omega_{,} \\ \sigma(\mathbf{u}) \cdot \mathbf{n}=\boldsymbol{\tau}, & \text { on } \Gamma_{N} \\ \mathbf{u}=\mathbf{0}, & \text { on } \Gamma_{0} \\ \mathbf{u}=\mathbf{u}_{\mathbf{D},}, & \text { on } \Gamma_{\mathbf{D}}\end{cases}
$$

where $\operatorname{div}(\cdot)$ is the divergence of a vector, $\mathbf{f}$ is the body force vector defined on mesh vertices, $\tau$ is the exerted external force on the boundary $\Gamma_{N}$ of outer normal direction $\mathbf{n}, \mathbf{u}_{\mathbf{D}}$ is the preset displacement field on boundary $\Gamma_{\mathbf{D}}$ and $\sigma$ is the stress tensor defined following the generalized Hooke's Law:

$$
\sigma=\mathrm{C} \epsilon
$$

Here $\mathbf{C}$ is the elasticity tensor of the material, $\epsilon$ is defined using $\mathbf{u}$ and the geometric deformation function

$$
\boldsymbol{\epsilon}=\frac{1}{2}\left(\nabla \mathbf{u}+\nabla \mathbf{u}^{T}\right)
$$

For the isotropic cases focused in this paper, $\mathbf{C}$ can be decomposed as follows:

$$
\mathbf{C}=\lambda \mathbf{I} \otimes \mathbf{I}+2 \mu \mathbf{I}
$$

where $\mathbf{I}$ is the second-rank identity tensor, $\operatorname{tr}(\cdot)$ is the trace of a matrix, $\lambda$ and $\mu$ are the Lame constants.

The Lame constants $\lambda$ and $\mu$ are related to the Young's modulus $E$ (a measure of stretch resistance) and Poisson's ratio $v$ (a measure of incompressibility) below:

$$
\mu=\frac{E}{2(1+v)}, \quad \lambda=\frac{E v}{(1+v)(1-2 v)} .
$$

For isotropic structures, only two independent variables are present in the matrix $\mathbf{C}$, i.e. $\{\lambda, \mu\},\{E, v\}$, or the matrix elements $\left\{C_{1111}, C_{1212}\right\}$.

\subsubsection{FEA to solve the equilibrium equations}

The linear elasticity (1) can be computed using the finite element method (FEM). First, $\Omega$ is discretized (Fig. 4(a)) into a set of volume elements, denoted as $\Omega_{\mathcal{M}}$ (Fig. 4(c)); the same as the discretization used for the semiregular structure generation in Section 3.1.1. When the domain is discretized, the physical fields, such as $\mathbf{u}$ and $\mathbf{f}$ are also discretized to vectors.

Then, the associated stiffness matrix $\mathbf{K}$ is assembled as follows:

$$
\mathbf{K}=\sum_{e \in \mathbf{e}} \mathbf{K}_{e}
$$

where $\mathbf{K}_{e} \in \mathbb{R}^{12 \times 12}$ is the associated submatrix to an element $e$ defined as follows:

$$
\mathbf{K}_{e}=\int_{\Omega} \nabla \boldsymbol{\phi}_{k}^{T} \mathbf{C}_{e} \nabla \boldsymbol{\phi}_{l} d \Omega,
$$

where $\mathbf{C}_{e}$ is the associated elasticity tensor related to the mesh element $e ; \boldsymbol{\phi}_{k} ; \boldsymbol{\phi}_{l}$ are the FE bases associated to the vertices of $e$.

After the above two steps, the problem of linear elasticity (1) is formulated as computing solution $\mathbf{u}$ to the following linear equation system in the matrix form:

$$
\mathbf{K u}=\mathbf{b}, \mathbf{u} \in \mathbb{R}^{d}
$$

where $d$ is the degree of freedoms of $\mathbf{u} ; \mathbf{u}$ and $\mathbf{b}$ are the vectors of the displacement solution and external loads.

\subsection{Problem statement}

As illustrated in Fig. 4, the problem of semiregular porous structure design is stated below: Find a proper radius distribution vector $\mathbf{r}$, i.e., to dig a hole of radius $r_{e}$ within each mesh element $e$ of $\Omega_{\mathcal{M}}$, so that the generated porous structure $\Omega_{\mathcal{M}, \mathbf{r}}$ has the specified displacement $\mathbf{u}_{D}$ along the boundary $\Gamma_{D}$ when an external force $\tau$ is applied on $\Gamma_{N}$.

In this problem, the mesh model $\Omega_{\mathcal{M}}$ is considered as a media model to generate the porous structure $\Omega_{\mathcal{M}, \mathrm{r}}$. The deformation behavior of $\Omega_{\mathcal{M}, \mathrm{r}}$ is similarly determined using (1), by replacing $\Omega$ with $\Omega_{\mathcal{M}, \mathbf{r}}$.

Consequently, the problem of semiregular porous structure design is casted into an optimization problem: 
To find a radius distribution vector $\mathbf{r}$ such that the deformation of $\Omega_{\mathcal{M}, \mathbf{r}}$ is close to the target deformation, i.e.,

$$
\min _{\mathbf{r}} d\left(\mathbf{r}, \mathbf{u}_{D}, \mathbf{u}\right),
$$

where $\mathbf{u}$ is the computed displacement to the linear elasticity problem in (1) for the porous structure $\Omega_{\mathcal{M}, \mathbf{r}}$, and $d\left(\mathbf{r}, \mathbf{u}_{D}, \mathbf{u}\right)$ measures the difference between the computed displacement $\mathbf{u}$ and specified displacement $\mathbf{u}_{D}$ :

$$
d\left(\mathbf{r}, \mathbf{u}_{D}, \mathbf{u}\right)=\left|\mathbf{u}-\mathbf{u}_{D}\right|_{\Gamma_{D}}
$$

\section{| $\cdot$ is the $L^{2}$ norm of a vector.}

Notably, only the voxelized model was studied here, i.e., models made of regular hexahedrons. The voxelized model can well approximate any free-form shape and be easily generated $[43,44]$. Thus the limitation does not restrict the problem studied. Such an approximation requires a mesh refinement, and adds more design freedoms, but the applied model reduction technique will reduce this design space.

Moreover, the porous model is created by digging a hole within each single hexahedral element. The holes are assumed to be symmetric with respect to $\mathrm{X}-\mathrm{Y}, \mathrm{Y}-\mathrm{Z}$, $Z-X$ planes, centering at the center of each hexahedral element. In this manner, one parameter can be used to describe the variation in the shape, and the homogenization theory can also be applied directly. Free-form shapes made of general cell elements will be studied in the future.

\subsection{Approach Overview}

The design optimization problem (9) is computationally expensive to directly solve considering the nonlinearity, a large number of design variables, and the fact that each optimization iterative step involves an expensive FE computation; see also Fig. 4. It will further add computational efforts because the volume mesh similar to that shown in Fig. 4(d) is regenerated during each iteration step because different design variables $\mathbf{r}$ will produce different computational domains.

An efficient optimization approach is proposed in this paper to overcome these challenges. See also Fig. 3. It essentially expresses the displacement of the design model as an analytical function in terms of the design variables. Novelty and good performance are mainly achieved via two main strategies:

1. Build the structure-property relationship, which relates a hole's radius to the quad-element's elasticity tensor,

2. Build the property-performance relationship, which relates the radius distributions to the deformation behavior of the target design.

This is achieved by the following three main techniques: the dimension reduction method, homogenization theory and combined approximation technique.

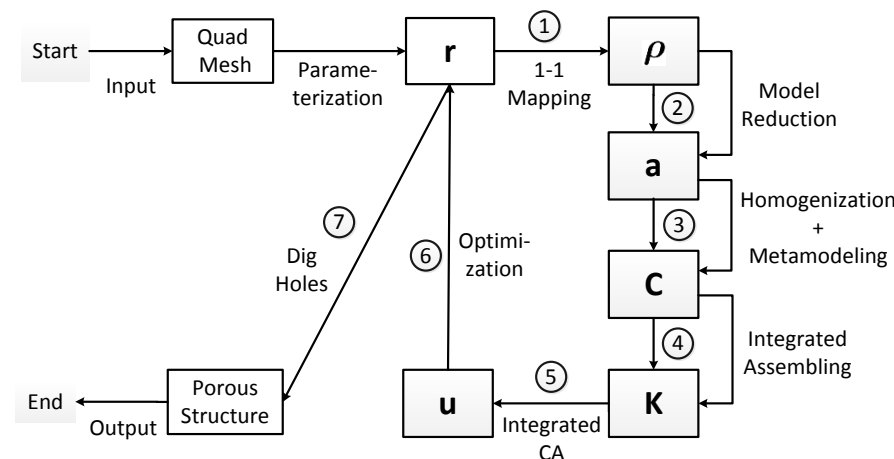

Figure 3: Flowchart of biscale design of a semiregular porous structure for the desired deformation behaviors, where $\mathbf{r}$ is the radius distribution, $\rho$ is the density distribution, $\mathbf{a}=\left\{a_{k}\right\}_{k=0}^{n}$ is the design coefficients after model reduction, $\mathbf{C}$ is the elasticity tensor, $\mathbf{K}$ is the derived stiffness matrix associated to a given structure, and $\mathbf{u}$ the computed displacement solution. The efficiency of the proposed approach is ultimately achieved by expressing the displacement of the design model as a function in terms of the design variables. Various techniques used to implement this strategy are also shown in the figure, including homogenization and metamodeling to build structure-property relationship, CA approximation to build property-performance relationship, and a model reduction technique to reduce the number of design variables.

The first two strategies build the structure-property relationship, and the last strategy builds the propertyperformance relationship. The derived analytical relationship avoids the complex FE analysis and mesh regeneration during each iteration step. Consequently, the original problem of finding the optimal radius distribution becomes a simple optimization problem without PDE constraints, and is very efficient to solve.

The strategy is implemented using an overall framework illustrated in Fig. 3 and includes seven main steps (Steps $1 \sim 7$ in the figure),

- Step 1: build the radius-density relationship.

- Step 2: build the density bases via model reduction, and convert design of the model's density distribution into an optimization problem in a much lower design space of finding the optimal coefficients to the density bases.

- Step 3: compute the elasticity tensors for a set of sample densities via a homogenization theory, and then build their analytical approximation via a metamodeling technique.

- Step 4: assemble the stiffness matrix using the above derived elasticity tensors for each mesh element, which gives the stiffness matrix in an analytical form with respect to the density coefficients.

- Step 5: the CA technique is further introduced to approximate the displacement solution difference due to the elasticity tensor difference, and thus an analytical function in terms of the design variables is derived. 


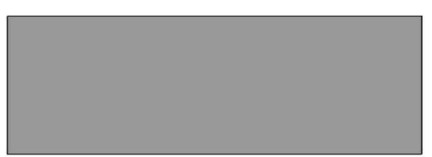

(a) Design area $\Omega$

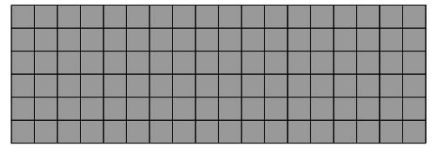

(c) Quad mesh of the design area (d) Mesh of porous structure for $\Omega_{\mathcal{M}}$

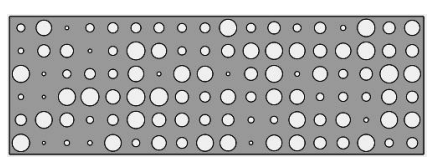

(e) Semiregular porous structure $\Omega_{\mathcal{M}, \mathbf{r}}$

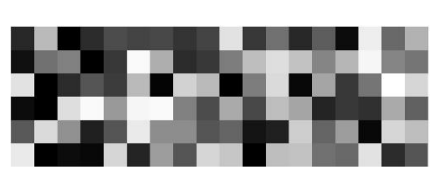

(g) Density distribution $\Omega_{\mathcal{M}, \rho}$

(b) FEA of solid model

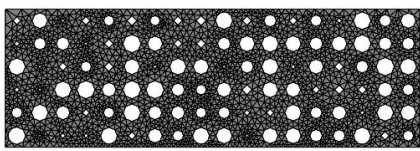
FEA

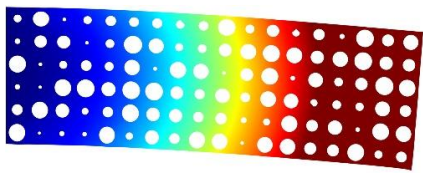

(f) FEA of porous structure

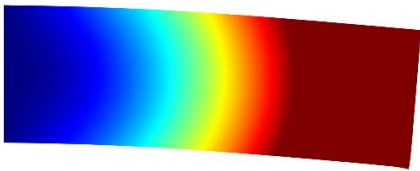

(h) FEA of homogenized porous

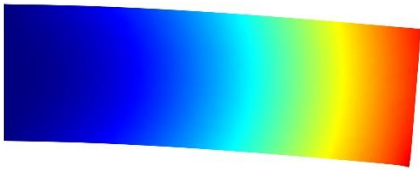
structure

Figure 4: Geometric models and simulation results.

- Steps 6,7: a generic optimization approach is applied to compute the optimal density coefficients, and correspondingly the radius distributions, affording the optimal semiregular porous structures.

Considering the example model shown in Fig. 2, various geometric models and simulation results are plotted in Fig. 4. The solid model $\Omega$, its associated quad mesh $\Omega_{\mathcal{M}}$, one of the porous structure $\Omega_{\mathcal{M}, \mathbf{r}}$ built from $\Omega_{\mathcal{M}}$, and the associated density model $\Omega_{\Omega, \rho}$ are shown in Figs. 4(a),(c),(e), and (g), respectively. The associated simulation results are shown in Figs. $4(\mathrm{~b}),(\mathrm{f})$, and (h), respectively, and the mesh model for $\Omega_{\mathcal{M}, \mathbf{r}}$ is shown in Fig. 4(d). Direct computation of the simulation result for model $\Omega_{\mathcal{M}, \mathbf{r}}$ in (e) built on its mesh model (d) is computationally expensive, whereas simulation of the property based on model $\Omega_{\Omega, \rho}$ will be much inexpansive. The proposed approach is based on such observations.

The technical details are explained below.

\section{Structure-property relationship}

In this section, the structure-property relationship that relates a porous element's design parameters to its elasticity tensor was first built by considering the fact that the number of design parameters is as large as the number of mesh elements. A model reduction approach was applied here, confining the design space to a linear space that significantly reduced the design parameters. To achieve this, the circular radius of an element was first converted to the corresponding density. Then, the homogenization simulation was applied to compute the relationship between each sampled design density with its associated elasticity tensor. Based on this, a metamodeling approach was used to build an approximate analytical structure-property relationship. The details are now explained.

\subsection{Dimension reduction of microstructure design space}

Before we start, one parameter was assigned to each cell, i.e., density $\rho$. As the cell had the size $1 \times 1$ in the $2 \mathrm{D}$ space and $1 \times 1 \times 1$ in the $3 \mathrm{D}$ space, with the round hole centered at the element's center, the round hole radius $r<$ 0.5 should describe the degree of freedom of the cell well. For reducing the power of the analytical expression of $\mathbf{u}$ derived later, density $\rho$ was introduced along with oneto-one mapping to $r$,

$$
\rho= \begin{cases}1-\pi r^{2}, & \text { in 2D space, } \\ 1-\frac{4}{3} \pi r^{3}, & \text { in 3D spcae }\end{cases}
$$

and in this sense, the density distribution describes all the degrees of freedom and is selected as the design parameters. However, the density distribution is also with high dimensions for complex structures.

The model reduction technique, as widely used in image processing, maps the image pixel space to the new space formed by a set of selected principle bases, thus significantly reducing the variable number, from the number of pixels to a few base coefficients. Inspired by this powerful capability, the design area was considered as an image pixel space by giving a "pixel" value (i.e., density) to each cell, and then the model reduction method was used similarly. As the image libraries are not available, the bases were derived by computing the eigenvectors to the following Laplacian eigenvalue problem:

$$
\nabla^{2} \rho_{j}=-\lambda_{j} \rho_{j}
$$

where $\nabla^{2}$ is the Laplacian operator, $\lambda_{j}$ is the $j$-th eigenvalue, and $\rho_{j}$, a vector of dimension $n$, is the associated eigenvector.

We then define the material base density set as follows:

$$
\left\{\boldsymbol{\rho}_{1}, \ldots, \boldsymbol{\rho}_{i}, \ldots, \boldsymbol{\rho}_{N}\right\}
$$

where $\rho_{i}$ is the eigenvector of the $i$-th largest eigenvalues, and $N$ is the number of selected bases with $N \ll n$.

Consequently, we define the target density distribution $\rho$ over the design area as the linear combinations of these eigenvectors, that is,

$$
\boldsymbol{\rho}=\boldsymbol{\rho}_{0}+\sum_{k=1}^{N} a_{k} \boldsymbol{\rho}_{k^{\prime}}
$$

where $\rho_{0}$ is a preset unified density distribution, and $a_{k}$ are the coefficients to be determined. 


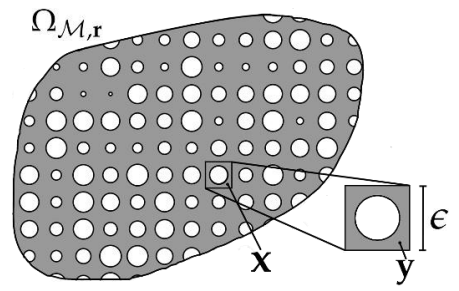

Figure 5: The macro- and micro- scale in the homogenization theory.

Note that some elements in $\rho_{i}$ may be negative. However, the elements of $\rho$ would not be negative in practice as the elements of $\rho_{0}$ are sufficiently positive and large to prevent negative values, with further constraints on coefficients $-1 \leq a_{k} \leq 1, k=1,2, . ., N$.

\subsection{Structure-property relationship via homogenization}

Given a density model $\Omega_{\mathcal{M}, \rho}$, here, we hope to determine the associated elasticity tensor $\mathbf{C}$ of each element in terms of its density, or building the structureproperty relationship. This relationship was achieved here with the classical homogenization approach [46], which computes the elasticity tensor for each sampled radius, and the metamodeling technique, which ultimately builds an approximate analytical structure-property relationship based on the computed results for the sampled cell.

\subsubsection{Homogenized elasticity tensor}

The homogenization theory essentially aims to calculate an average material property for each cell element. In this study, the area of the cell was assumed to be relatively small enough so that it can be seen as a homogenized point in the macroscopic scale, filled with evenly distributed virtual material. In this sense, each virtual material's elasticity property was characterized by a homogenized elasticity tensor, describing the elastic behavior of each cell in the area $\Omega$ of the macroscale; see also the stiffness matrix assembly process involved in (6) and (7).

We now explain details of computing the elasticity tensor using the homogenization theory. Consider a porous structure $\Omega_{\mathcal{M}, \mathbf{r}}$; see also Fig. 5 . Let $e$ be the $\mathbf{X}$-th cell with respect to $\Omega_{\mathcal{M}}$, or the cell's coordinate in the macroscale, and $\epsilon$ the size of the cell. Let $\mathbf{y}$ be the coordinate of a point inside $e$ in the microscale, and $\mathbf{x}$ the coordinate of the point in the microscale. Correspondingly, we have the association between the micro- and macro- coordinates

$$
\mathbf{x}=\mathbf{X}+\mathbf{y}, \quad 0 \leq \mathbf{y} \leq \epsilon
$$

For the specific linear elasticity problem studied here, the homogenization theory aims to approximate the elasticity tensor $\mathrm{C}^{H}(e)$ for cell $e$ via equalizing their potential energy at the macro- and micro- scales. This classical theory is well studied as shown in the following result:
Lemma 1. [46] Let $C_{i j p q}(e, \mathbf{y})$ be the elasticity tensor at every micropoint $\mathbf{y}$ for a cell e located at $\mathbf{X} ; C_{i j p q}(e, \mathbf{y})$ takes zero for void material. For each specific pair of $1 \leq i, j, k, l \leq 2$, the homogenized elasticity tensor $C_{i j k l}^{H}(e)$ can be approximated as

$$
C_{i j k l}^{H}(e)=\frac{1}{|e|} \int_{e}\left(C_{i j k l}(\mathbf{X}, \mathbf{y})-C_{i j p q}(\mathbf{X}, \mathbf{y}) \frac{\partial \mu_{p}^{k l}}{\partial \mathbf{y}_{q}}\right) d \mathbf{y},
$$

where the virtual displacements $\mu_{p}^{k l}$ is obtained via solving the following problem,

$$
\int_{e}\left(C_{i j k l}-C_{i j p q} \frac{\partial \mu_{p}^{k l}}{\partial \mathbf{y}_{q}}\right) \frac{\partial \mathbf{v}_{1 i}}{\partial \mathbf{y}_{j}} d \mathbf{y}=0,
$$

where $\mathbf{v}_{1 i}$ denotes one arbitrary micro-displacements, and the product here means the Einstein Notation, as the summation of the products under all the possible indexes.

Note that the above lemma applies to every pair of $(i, j, k, l)$, but we only need to compute two specific elements in $\mathbf{C}^{H}$ for isotropic geometries, because $\mathbf{C}^{H}(\mathbf{X})$ only has two independent variables.

\subsubsection{Metamodeling}

Metamodeling is a widely-used method to model complex relations. For example, Ghasemi et al. [29, 30, 31] used metamodeling to capture uncertainties in multiscale modeling for complex structures like fiber-reinforced structures. In this study, based on the homogenization theory in Lemma 1, for a set of sample cells of different density $\rho$, the elasticity tensors were separately computed and denoted with $\rho$ as $\mathbf{C}(\rho)$. Then following the GibsonAshby model [47], a second-order polynomial function was used to build their approximation,

$$
\mathbf{C}(\rho)=\mathbf{C}_{0}+\mathbf{C}_{1} \rho+\mathbf{C}_{2} \rho^{2},
$$

where $\mathbf{C}_{0}, \mathbf{C}_{1}, \mathbf{C}_{2}$ are constant elasticity tensors with respect to $\mathbf{C}$.

The numerical approach to build the structureproperty relationship using the metamodeling technique is explained below.

Inputs: A unit cell $\mathbf{X}$ of elasticity tensor $\mathbf{C}$ in the $\mathrm{mi-}$ croscale determined by its material;

Outputs: The analytical density-elasticity relationship in a form as shown in (18) for cell $X$.

1. Sample uniformly a set of different pore radii $r$ of a unit cell.

2. For each $r$, calculate its corresponding elasticity tensor $C_{1111}, C_{1212}$ based on Lemma 1 as follows:

- Compute the virtual displacement $\mu_{p}^{k l}$ by solving (17) as a local linear elasticity problem defined in cell $\mathbf{X}$ using FEA.

- Compute the effective elasticity tensor $C_{i j k l}^{H}$ by substituting solution $\mu_{p}^{k l}$ into (16). 
3. For each $r$, map $r$ to its corresponding $\rho$ via (11). Build the approximation relation $C_{1111}(\rho), C_{1212}(\rho)$ in the form as (18) using the results from Step 2. Other elements in $\mathbf{C}(\rho)$ can also be derived.

\section{Property-performance relationship}

Once the elasticity tensor $\mathbf{C}$ for each design cell $\mathbf{X}$ is derived analytically in terms of the density coefficients, an analytical relationship between the microstructure and the target displacement was further derived in this section.

The analytical relationship is mainly achieved based on the following observations. Different design parameters (i.e. density base coefficients), produce different cell density distributions and thus their associated elasticity tensors. From the FE assembly process introduced in Section 3.1.4, different stiffness matrices are ultimately produced. The associated displacement solutions to the different stiffness matrices, can then be approximated by the newly introduced combined approximation (CA) technique. The details are as follows:

\subsection{Stiffness matrix expansion}

From the model reduction process built in Section 4.1, various density distributions can be designed by changing the density base coefficients in the linear combination (14). In further combination with the FE method, a similar linear relationship also exists between the density base coefficients and their associated stiffness matrices as explained below.

Lemma 2. Let $\rho$ be a density distribution in a form as (14), and $\mathbf{K}$ the stiffness matrices for $\boldsymbol{\rho}$. We have

$$
\mathbf{K}=\mathbf{K}_{0}+\triangle \mathbf{K}
$$

where

$$
\triangle \mathbf{K}=\sum_{k=0}^{N} a_{k} \mathbf{K}_{k}+\sum_{m=0}^{N} \sum_{n=0}^{N} a_{m} a_{n} \mathbf{K}_{m n},
$$

and $\mathbf{K}_{0}, \mathbf{K}_{k}, \mathbf{K}_{m n}$ are assembled cell by cell using $\mathbf{K}_{0, e}, \mathbf{K}_{k, e}$, $\mathbf{K}_{m n, e}$, which are associated to $\boldsymbol{\rho}_{0}, \boldsymbol{\rho}_{k}, \boldsymbol{\rho}_{m} \boldsymbol{\rho}_{n}$ as follows:

$$
\begin{gathered}
\mathbf{K}_{0, e}=\int_{\Omega_{e}} \nabla \boldsymbol{\phi}_{i}^{T} \mathbf{C}_{0}^{\prime} \nabla \boldsymbol{\phi}_{j} d \Omega, \mathbf{K}_{k, e}=\int_{\Omega_{e}} \nabla \boldsymbol{\phi}_{i}^{T} \mathbf{C}_{1}^{\prime} \rho_{k} \nabla \boldsymbol{\phi}_{j} d \Omega, \\
\mathbf{K}_{m n, e}=\int_{\Omega_{e}} \nabla \boldsymbol{\phi}_{i}^{T} \mathbf{C}_{2}^{\prime} \rho_{m} \rho_{n} \nabla \boldsymbol{\phi}_{j} d \Omega .
\end{gathered}
$$

Proof: Given in Appendix A.

\subsection{State solution relation}

Once the relationship between the density base coefficients and stiffness matrices is built, their corresponding state solutions can then be related as well. Specifically, the solution $\mathbf{u}$ can be expressed in an analytical form with respect to the coefficients $\mathbf{a}=\left\{a_{k}\right\}_{k=0}^{N}$. This advantage is obtained by introducing the approach of the combined approximation (CA), first introduced in structure reanalysis [32]. The CA approach combines the Taylor series expansion with the reduced base approach, taking base vectors as the terms in the binomial series. For low-rank and moderately high-rank modifications to structures, the CA approach can produce the solution approximation, both accurate and computationally efficient.

Combining results from Lemma 2 and (8), we have

$$
\mathbf{K}_{0} \mathbf{u}_{0}=\mathbf{f}, \quad \mathbf{K u}=\left(\mathbf{K}_{0}+\triangle \mathbf{K}\right) \mathbf{u}=\mathbf{f},
$$

where $\mathbf{u}_{0}, \mathbf{u}$ are state solutions, and $\triangle \mathbf{K}$ is linearly correlated with a.

Following the result of CA directly, the following results were obtained on approximating $\mathbf{u}$ using solution $\mathbf{u}_{0}$.

Lemma 3. [32] We have the following approximation solution for (22),

$$
\mathbf{u}=\mathbf{u}_{0}-\sum_{i=1}^{t} q_{i} \mathbf{u}_{i}=\mathbf{u}_{0}-\mathbf{D} \mathbf{q}
$$

where each $\mathbf{u}_{i}$ is iteratively defined as follows

$$
\mathbf{K}_{0} \mathbf{u}_{i}=-\triangle \mathbf{K} \mathbf{u}_{i-1}, \quad i=1, \ldots, t,
$$

and the coefficients $\mathbf{q}$ are determined by solving the following reduced system

$$
\mathbf{D}^{T}(\mathbf{K}+\triangle \mathbf{K}) \mathbf{D q}=\mathbf{D}^{T} \triangle \mathbf{K u}_{0} .
$$

where the matrix $\mathbf{D}$ is a collection of $\mathbf{u}_{i}$ as shown in (23).

As defined iteratively above, $\mathbf{u}_{i}$ is $i$-th order polynomial expression of parameter $\mathbf{a}$, and generally for saving computational costs, $\mathbf{q}$ was set as the all-one vector. Therefore, the order of the polynomial approximation $\mathbf{u}(\mathbf{a})$ will be $t$.

\subsection{Optimization}

Based on the above analysis, the design of porous structures satisfying a certain displacement requirement $\mathbf{u}_{\mathbf{D}}$ is as follows:

Theorem 1. Solution to the minimization problem in (9) can be obtained by solving the following minimization problem:

$$
\min _{\left\{a_{k}\right\}}\left|\mathbf{u}_{0}-\mathbf{D q}-\mathbf{u}_{\mathbf{D}}\right|
$$

where

$$
\begin{gathered}
\mathbf{D}=\left[\mathbf{u}_{1} \mathbf{u}_{2} \ldots \mathbf{u}_{t}\right], \quad-1 \leq a_{k} \leq 1, k=1,2, \ldots, N \\
\boldsymbol{\rho}=\boldsymbol{\rho}_{0}+\sum_{k=1}^{N} a_{k} \boldsymbol{\rho}_{k} .
\end{gathered}
$$

Notably, the optimization problem defined in (26) is very different from the optimization problem defined in (9). The latter has a polynomial goal with linear constraints, and is very easy to solve, whereas the former expression is explicit in its variables $\left\{a_{k}\right\}_{k=1}^{N}$. Many existing sophisticated algorithms can handle this problem, such as BFGS [48] (Broyden Fletcher Goldfarb Shanno algorithm, a Quasi-Newton Method). 


\section{Performance Test}

The proposed approach was implemented using COMSOL Multiphysics ${ }^{\circledR}$ and Matlab on a computer with Intel Core i3-2100 CPU. Various aspects of the proposed approach were tested. In all the tests, a regular planar rectangular of size $30[\mathrm{~mm}] \times 10[\mathrm{~mm}]$ was taken. The model was made of a material of Young's modulus $E=50[\mathrm{MPa}]$ and Poisson's ratio $v=0.3$. For the first three examples, the model was fixed along its left side, and pulled at its right bottom point of a load of $-1[k N]$. The goal was to design a cellular structure so that the right-bottom point has a desired displacement; the fourth will be explained later. In all the tests, 12 eigen-bases were selected in the model reduction process, as they demonstrate a good balance between computational accuracy and efficiency.

\subsection{Accuracy test of analytical expression}

The derived analytical displacement expression in (23) forms the base of the proposed approach, and its accuracy determines that of the final result. Thus, the simulation results based on (23) were first compared with those derived by the homogenization approach and those by the direct FE analysis.

The approach works similarly for other cases of holes, and four different hole cases were tested here: random distributed circular holes, square holes, hexagon holes, or cross holes, as shown below and in Table 2 from the left to the right.

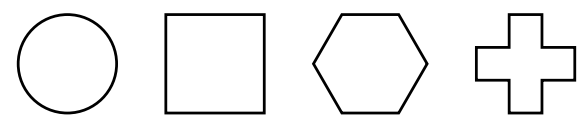

In these tests, the maximal inscribed circle, square, or hexahedron was first built, and then each cellular hole was scaled at different factors ranging from 0.3 to 0.9 for testing.

Appendix B shows the concrete analytical expression to (23) for the case of circular holes for a concrete impression of the analytic displacement expression.

The test results are summarized and compared in Table 2. The first row shows the computed micro-structures and their deformations at the scaling factor 0.5 . The second row compares the computed displacement of the right-bottom point derived by the three different approaches. They demonstrate close approximation.

Table 3 also compares the maximal simulation errors between the FEA and analytical solution, between the FEA and the homogenization, and between the homogenization and the analytical solution, known as $\mathrm{A}$-error, $\mathrm{H}$ error and $H A$-error, respectively. The results indicate the final A-error was $\sim 12.0 \%$, and is acceptable. Notably, the HA-error ranges from $2.9 \%$ to $5.6 \%$, and is much smaller than the H-error, ranging from $8.1 \%$ to $17.8 \%$. This tells us that the approximation A-error is mainly due to the homogenization process (H-error), and the CA approximation (HA-error) is much smaller compared to it. Both of them contribute to the A-error. More accurate homogenization technique may further improve the simulation accuracy. The table also shows that the larger the hole sizes, the larger the error caused by the approximation. This is probably because the homogenization theory works better for cells of smaller sizes.

\subsection{Grid resolution influence test}

The derived porous structure is dependent on the resolution of the prefixed regular quad-mesh. We test here the impacts of the grid resolution on the final results of porous structures. In this test, the design area size, the external loadings are kept the same as before, and the grid resolutions are varied differently, respectively, $12 \times 4$, $18 \times 6,24 \times 8$ and $30 \times 10$. The four derived optimal porous structures and their corresponding pore size distributions are shown in Fig. 6.

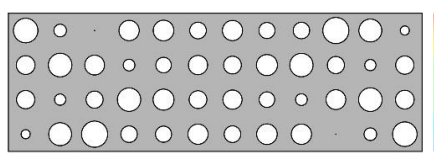

(a) $12 \times 4$

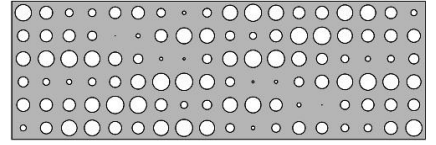

(c) $18 \times 6$

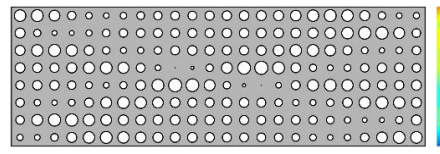

(e) $24 \times 8$

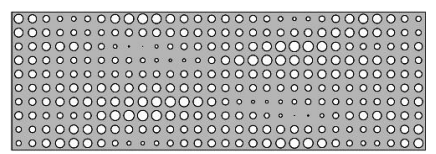

(g) $30 \times 10$

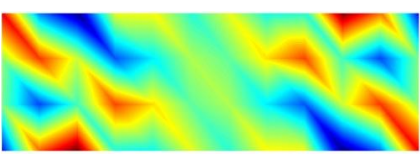

(b) $12 \times 4$ (Colormap)

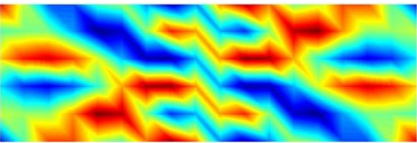

(d) $18 \times 6$ (Colormap)

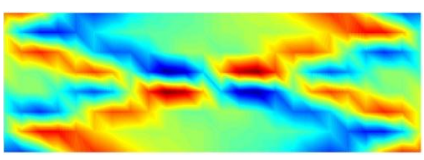

(f) $24 \times 8$ (Colormap)

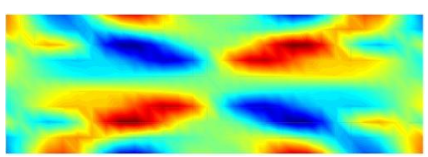

(h) $30 \times 10$ (Colormap)
Figure 6: Microstructures and the corresponding distribution colormaps generated under four different grid resolutions: (a) $12 \times 4$; (c) $18 \times 6$; (e) $24 \times 8$; $(\mathrm{g}) 30 \times 10$. The colormaps (jet) show the hole radius distribution, red corresponding to the larger radius and blue corresponding to the smaller radius.

As can be observed from the results, different grid resolutions produce different pore sizes within the design domain. However, the size distributions are very similar, as can also be observed from the colormap in Fig. 6. It tells us that the proposed approach is quite independent of the grid resolutions, based on which numerical coarsening and refining is feasible for different design purposes. We also notice that as higher resolutions are applied, more detailed holes may appear in the final design, and the design target is approximated more accurately as seen from Table 4. 
Table 2: Test of simulation accuracy of four different porous structures.
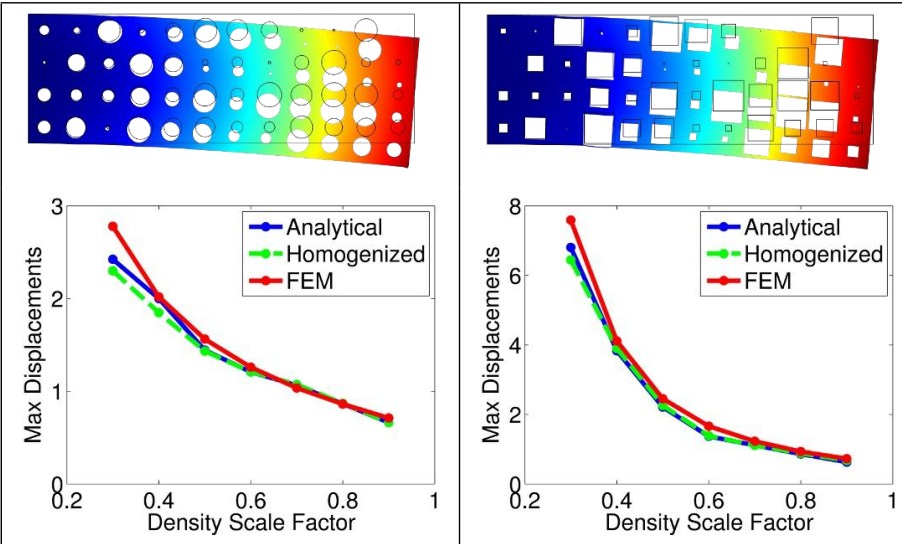

Table 3: Maximal error comparisons of the four different cases in Table 2: (a) A-error: error between the FEA and analytical simulation; (b) H-error: error between the FEA and homogenization; (c) HA-error: error between the homogenization and analytical solution.

\begin{tabular}{c|c|c|c} 
Sample Approach & A-error & H-error & HA-error \\
\hline Circular & $12.8 \%$ & $17.2 \%$ & $-5.1 \%$ \\
Square & $13.0 \%$ & $9.9 \%$ & $2.9 \%$ \\
Hexagon & $11.5 \%$ & $17.8 \%$ & $-5.6 \%$ \\
Cross & $11.4 \%$ & $8.1 \%$ & $3.0 \%$ \\
\hline
\end{tabular}

Table 4: Percentages of maximal displacement error under different resolutions between the targets and results after the design.

\begin{tabular}{c|c|c|c|c}
\hline Error & $12 \times 4$ & $18 \times 6$ & $24 \times 8$ & $30 \times 10$ \\
\hline Error PCT. & $14.6 \%$ & $11.2 \%$ & $10.3 \%$ & $9.7 \%$ \\
\hline
\end{tabular}

\subsection{Computational efficiency tests}

Computational efficiency is one of the main contribution made in this study. Its efficiency was further stated by comparing its running times with that of directly solving (9) by MMA (Moving Asymptotes Method) [49], a classical optimization approach widely used in topology optimization.

\subsubsection{Design under varying resolutions}

The proposed approach was tested on a design domain with different numbers of mesh elements, i.e., $12 \times 4$, $18 \times 6,24 \times 8,30 \times 10,80 \times 20,120 \times 30$ and $200 \times 50$. The comparison results are summarized in Table 5. As shown from the left part of Table 5, the overall speed-up ranged from 70.9 to 179.1 times. Furthermore, as the resolutions increased, the number of iterations increased significantly in the MMA approach, but almost remained the same in our approach, demonstrating the proposed approach's nice convergence. This was also observed in the plot of convergence curve in Fig. 7 for the $18 \times 6$ mesh.
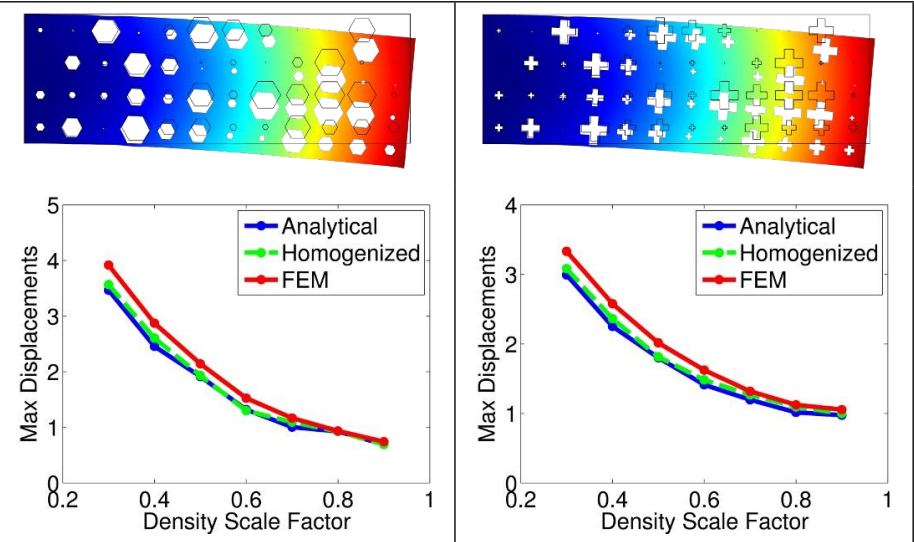

We also compare the error relative to the target displacement in each critical iterative step of our approach and MMA in Table 6. The two approaches start from the same evenly distributed structure, evolves close to the preset target. In this case, our approach has a significant advantage in the convergence speed and a small advantage in the final error control.

\subsubsection{Design under varying targets}

The efficiency of the proposed approach was further tested in designing porous structures under different design targets using the same models used as above. The target right-bottom point displacements were sampled 10 times in the range $0.1-0.3[\mathrm{~mm}]$ in the $Y$ direction.

The numerical results are summarized in the right part of Table 5. Compared to the time cost for one design target, the proposed approach costs almost the same time while MMA takes almost 10 times. Taking the $12 \times 4$ as an example, the computational cost of our solver increased from $0.11[s]$ to $0.21[s]$, whereas MMA increased the cost from 8.31[s] to 83.1[s]. Further study showed that the time cost in computing the analytical expression (23) is $\sim 0.10[s]$, whereas the optimization step costs only $\sim 0.01[s]$, which is much smaller.

These results indicate that the proposed approach is very efficient in porous structure design mainly because of its derived analytical displacement expression shown in (23). Such a nice expression will be used in each optimization iteration step and also many times for different design targets. In both the cases, the analytical expression can only be computed once, involving one step of expensive FE computations, and other steps of optimization are almost computationally negligible.

\subsection{Multitarget design}

The ability of the proposed approach in meeting multiple displacement targets was tested. The example shown in Fig. 8(a) consisted of $18 \times 6$. Both the left and right sides of the model are fixed, and a load of $-10 \mathrm{kN} / \mathrm{m}^{2}$ was exerted on its top. We aimed to design a porous 
Table 5: Algorithm efficiency comparison with MMA in both single and multiple editing situations.

\begin{tabular}{c|c|c|c|c|c||c|c|c|c}
\hline Model & Our Solver & Iter. & MMA & Iter. & Speed-up & $\begin{array}{c}\text { No. of } \\
\text { Editing }\end{array}$ & $\begin{array}{c}\text { Our Solver } \\
\text { (Total) }\end{array}$ & $\begin{array}{c}\text { MMA } \\
\text { (Total) }\end{array}$ & $\begin{array}{c}\text { Speed-up } \\
\text { (Total) }\end{array}$ \\
\hline $12 \times 4$ & $0.11 \mathrm{~s}$ & 5 & $8.31 \mathrm{~s}$ & 255 & $75.5 \times$ & 10 & $0.21 \mathrm{~s}$ & $83.1 \mathrm{~s}$ & $395.7 \times$ \\
$18 \times 6$ & $0.18 \mathrm{~s}$ & 4 & $12.77 \mathrm{~s}$ & 350 & $70.9 \times$ & 10 & $0.28 \mathrm{~s}$ & $127.7 \mathrm{~s}$ & $456.1 \times$ \\
$24 \times 8$ & $0.25 \mathrm{~s}$ & 6 & $32.29 \mathrm{~s}$ & 853 & $129.2 \times$ & 10 & $0.35 \mathrm{~s}$ & $322.9 \mathrm{~s}$ & $922.6 \times$ \\
$30 \times 10$ & $0.57 \mathrm{~s}$ & 4 & $102.1 \mathrm{~s}$ & 3122 & $179.1 \times$ & 10 & $0.67 \mathrm{~s}$ & $1021 \mathrm{~s}$ & $1523.9 \times$ \\
$80 \times 20$ & $8.70 \mathrm{~s}$ & 5 & $888.3 \mathrm{~s}$ & 8595 & $102.1 \times$ & 10 & $8.8 \mathrm{~s}$ & $8883 \mathrm{~s}$ & $1009.4 \times$ \\
$120 \times 30$ & $29.60 \mathrm{~s}$ & 6 & $4716.9 \mathrm{~s}$ & 15723 & $159.4 \times$ & 10 & $29.7 \mathrm{~s}$ & $47169 \mathrm{~s}$ & $1588.2 \times$ \\
$200 \times 50$ & $103.1 \mathrm{~s}$ & 5 & $16626.5 \mathrm{~s}$ & 33251 & $161.3 \times$ & 10 & $103.2 \mathrm{~s}$ & $166265 \mathrm{~s}$ & $1611.1 \times$ \\
\hline
\end{tabular}

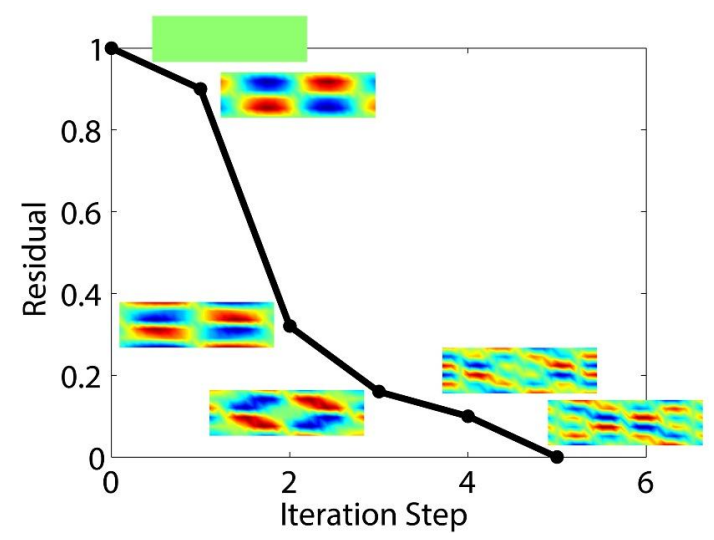

(a) Convergence curve using our approach

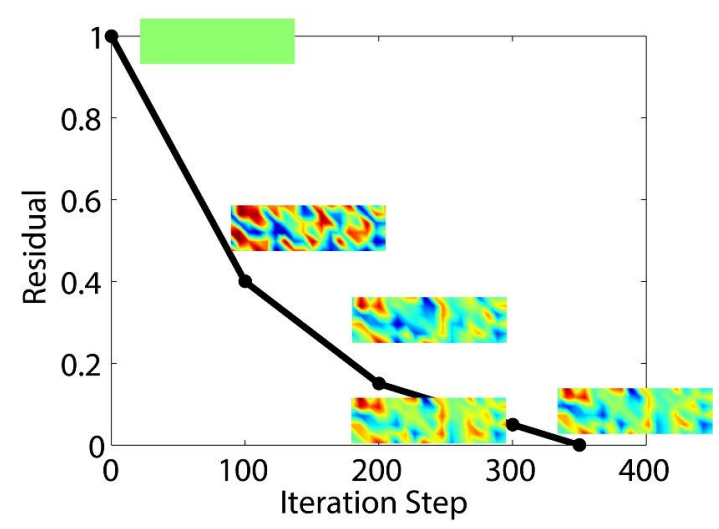

(b) Convergence curve using MMA

Figure 7: Convergence speed comparison between MMA and the proposed approach when solving the same problem.

structure that deforms to fit a preset cubic Bézier curve with control points $C P_{1}, C P_{2}, C P_{3}$, and $C P_{4}$. Three tests were performed: We fixed $C P_{1}=(0,0), C P_{4}=(30,0)$, $C P_{2}=(c y, 20)$, and $C P_{3}=(c y, 30)$ for different $c y=$ $-0.08,-0.16,-0.24$. In these tests, a set of 18 sampling points were selected at the bottom of the test beam, so that they will approximate the target Bézier curve respectively. For each test case, the computed porous structures using the proposed approach and its deformation are plotted in Fig. 8(b), (c) and (d). The deformation was also compared to their corresponding Bézier curve, and they all showed close shape approximation.
Table 6: Error relative to the target displacement in each critical iterative step of our approach and MMA.

\begin{tabular}{c|c|c|c|c|c}
\hline Ours Step & 1 & 2 & 3 & 4 & 5 \\
\hline Error & $40.2 \%$ & $35.2 \%$ & $16.8 \%$ & $14.0 \%$ & $11.3 \%$ \\
\hline SMA & 1 & 100 & 200 & 300 & 350 \\
\hline Error & $40.2 \%$ & $22.5 \%$ & $16.4 \%$ & $13.8 \%$ & $12.1 \%$ \\
\hline
\end{tabular}

\subsection{D example}

The performance of the proposed approach was also tested for the 3D complex automobile hubcap models shown in Fig. 9. In the test, the hubcap was made up of material " $A 356$ " of $E=6.9 \times 10^{4}[\mathrm{MPa}], v=0.33$, and $\rho=2690\left[\mathrm{Kg} / \mathrm{m}^{3}\right]$. The hubcap takes a volume of $0.8[\mathrm{~m}] \times 0.15[\mathrm{~m}] \times 0.8[\mathrm{~m}]$. The outer wheel rim has a radius of $0.4[\mathrm{~m}]$ and a width of $0.05[\mathrm{~m}]$ with no groove. The five ribs of the wheel have a width of $0.03[\mathrm{~m}]$. And the inner circle has a radius of $0.2[\mathrm{~m}]$ and the circle hole has a radius of $0.04[\mathrm{~m}]$.

The test was performed with the following physical constraints: The inner circle was fixed (in the area labeled blue), and an external load of sum $F=1081[N]$ was added averagely on the wheel rim (as shown by the arrows). The displacement (of the red point in the red box) was set as $0.2[\mathrm{~mm}]$.

The derived hole distribution is shown in Fig. 10; subtracting these holes from the original hubcap affords the final design. As observed from the hole distribution, the holes in the five ribs will be made relatively smaller than those in the wheel, indicating that the ribs provide the main resistance against the forces and must be stiffer. Moreover, the uneven hole distributions either in the outer rim or the inner circle show that some characters of PCA bases remain in the final result.

\section{Conclusions and future work}

Based on a novel concept and representation of a semiregular structure, the problem of biscale porous structure was studied for desired deformation behavior. Due to the usage of various advanced numerical techniques, the target displacement was ultimately expressed as a function in terms of the design variables, and thus the 


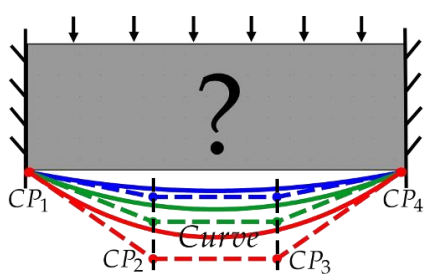

(a) Curve and Area

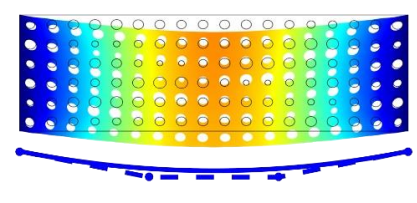

(b) Blue curve simulation

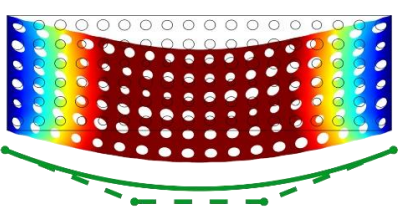

(c) Green curve simulation

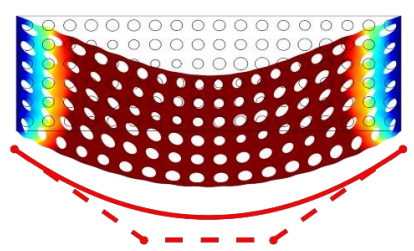

(d) Red curve simulation

Figure 8: Example of multitarget design: The cellular model was designed to fit three different types of Bézier curves under the same boundary condition.

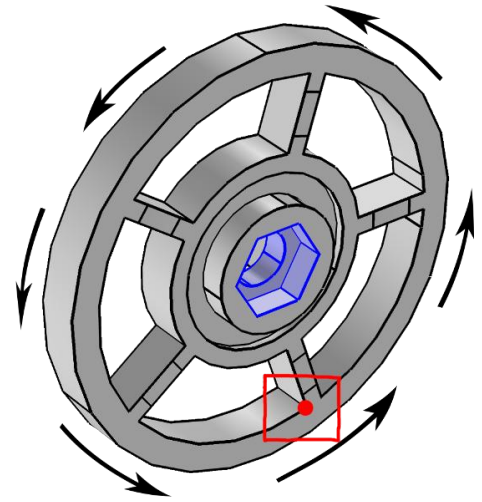

Figure 9: External forces (around the hubcap), fixed part (inner blue part) and focused point (the red point in the red box) of the automobile hubcap.

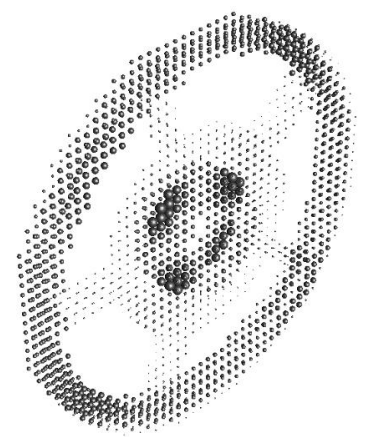

Figure 10: Inner structure of the voxelized automobile hubcap after our design approach. The final designed hubcap is the subtraction of the original hubcap and this inner structure. associated optimization problem were efficiently solved. Various numerical examples were also tested to demonstrate the approach's accuracy, dependency on mesh resolution and computational efficiency.

Limitations. For some complex structures, our approach can not fully approximate the design region, particularly with curve edges/surfaces. Although the area and effect of edges/surfaces are small, they still affect the overall performance. In the future, we plan to resolve this issue using homogenization method over an arbitrary hex mesh. Furthermore, the proposed approach mainly focused on a porous structure made by digging a isotropic hole within its interior, which may limit the range of the material's physical property and consequently the final design performance. In the future, other types of complex shapes will be studied for performance improvement. For such problems, the model reduction applied here may not work well, and other advanced model reduction techniques should be developed. In addition, the proposed approach is limited when being extended to microstructures other than porous structures, such as fiber-reinforced microstructures. We will follow the biscale flowchart and advance the homogenization method for the extension in the future.

\section{Appendix A: Proof to Lemma 2}

Proof: Consider a mesh element $e$ of $\Omega_{\mathcal{M}, \mathrm{r}}$. Let $\mathbf{C}_{e}$ be its elasticity tensor. Substituting the decomposition form (14) of the density $\rho$ into (18) gives

$$
\mathbf{C}_{e}(\rho)=\mathbf{C}_{0}+\mathbf{C}_{1}\left(\rho_{0}+\sum_{k=1}^{N} a_{k} \rho_{k}\right)+\mathbf{C}_{2}\left(\rho_{0}+\sum_{k=1}^{N} a_{i} \rho_{i}\right)^{2}
$$

Rearranging the above equation as an equation of variables $\mathbf{a}=\left\{a_{k}\right\}_{k=0}^{N}$ gives

$$
\mathbf{C}_{e}(\mathbf{a})=\mathbf{C}_{0}^{\prime}+\sum_{k=1}^{N} \mathbf{C}_{1}^{\prime} \rho_{k} a_{k}+\sum_{m=1}^{N} \sum_{n=1}^{N} \mathbf{C}_{2}^{\prime} \rho_{m} \rho_{n} a_{m} a_{n}
$$

where

$$
\mathbf{C}_{0}^{\prime}=\mathbf{C}_{0}+\mathbf{C}_{1} \rho_{0}+\mathbf{C}_{2} \rho_{0}^{2}, \mathbf{C}_{1}^{\prime}=\mathbf{C}_{1}+2 \mathbf{C}_{2} \rho_{0}, \mathbf{C}_{2}^{\prime}=\mathbf{C}_{2} .
$$


Then we have the sub-matrix $\mathbf{K}_{e}$ to element $\mathrm{e}$

$$
\begin{aligned}
\mathbf{K}_{e} & =\int_{\Omega_{e}} \nabla \boldsymbol{\phi}_{i}^{T} \mathbf{C}_{e}(\mathbf{a}) \nabla \boldsymbol{\phi}_{j} d \Omega \\
& =\mathbf{K}_{0, e}+\sum_{k=0}^{N} a_{k} \mathbf{K}_{k, e}+\sum_{m=0}^{N} \sum_{n=0}^{N} a_{m} a_{n} \mathbf{K}_{m n, e},
\end{aligned}
$$

where

$$
\begin{gathered}
\mathbf{K}_{0, e}=\int_{\Omega_{e}} \nabla \boldsymbol{\phi}_{i}^{T} \mathbf{C}_{0}^{\prime} \nabla \boldsymbol{\phi}_{j} d \Omega, \mathbf{K}_{k, e}=\int_{\Omega_{e}} \nabla \boldsymbol{\phi}_{i}^{T} \mathbf{C}_{1}^{\prime} \rho_{k} \nabla \boldsymbol{\phi}_{j} d \Omega, \\
\mathbf{K}_{m n, e}=\int_{\Omega_{e}} \nabla \boldsymbol{\phi}_{i}^{T} \mathbf{C}_{2}^{\prime} \rho_{m} \rho_{n} \nabla \boldsymbol{\phi}_{j} d \Omega .
\end{gathered}
$$

Considering the assembly process (6), we have

$$
\mathbf{K}=\mathbf{K}_{0}+\triangle \mathbf{K},
$$

for

$$
\triangle \mathbf{K}=\sum_{k} a_{k} \mathbf{K}_{k}+\sum_{m, n} a_{m} a_{n} \mathbf{K}_{m n}
$$

\section{Appendix B: the Analytical Displacement Expression}

Analytical displacement expression of (23) for the case of circular holes in in Section 6.1:

$u=-9.694+0.2139 a_{1}+0.2593 a_{2}-0.8453 a_{3}+$ $0.3508 a_{4}-1.614 a_{5}+0.2823 a_{6}-0.8797 a_{7}+0.2724 a_{8}-$ $4.732 a_{9}+0.3525 a_{10}+1.291 a_{11}+0.1932 a_{12}-0.01653 a_{1}^{2}-$ $0.02771 a_{2}^{2}+0.1036 a_{3}^{2}-0.03201 a_{4}^{2}+0.07688 a_{5}^{2}-$ $0.02419 a_{6}^{2}+0.1223 a_{7}^{2}-0.03089 a_{8}^{2}+2.367 a_{9}^{2}-$ $0.07833 a_{10}^{2}-0.04033 a_{11}^{2}-0.01743 a_{12}^{2}-0.01038 a_{1} a_{2}+$ $0.03219 a_{1} a_{3}-0.1227 a_{1} a_{4}+0.07268 a_{1} a_{5}-0.01284 a_{1} a_{6}+$ $0.03882 a_{1} a_{7}-0.01374 a_{1} a_{8}+0.9520 a_{1} a_{9}-0.01872 a_{1} a_{10}-$ $0.2889 a_{1} a_{11}-0.01017 a_{1} a_{12}+0.02379 a_{2} a_{3}-0.02019 a_{2} a_{4}+$ $0.8902 a_{2} a_{5}-0.01233 a_{2} a_{6}+0.03482 a_{2} a_{7}-0.02214 a_{2} a_{8}+$ $0.9721 a_{2} a_{9}-0.02561 a_{2} a_{10}-0.3122 a_{2} a_{11}-0.01312 a_{2} a_{12}+$ $0.2891 a_{3} a_{4}-0.7821 a_{3} a_{5}+0.02109 a_{3} a_{6}-0.7452 a_{3} a_{7}+$ $0.2844 a_{3} a_{8}-1.2771 a_{3} a_{9}+0.6722 a_{3} a_{10}+0.7913 a_{3} a_{11}+$ $0.01689 a_{3} a_{12}+0.5224 a_{4} a_{5}-0.02459 a_{4} a_{6}+0.05270 a_{4} a_{7}-$ $0.02894 a_{4} a_{8}+0.8719 a_{4} a_{9}-0.04876 a_{4} a_{10}-0.3779 a_{4} a_{11}-$ $0.02287 a_{4} a_{12}+0.2836 a_{5} a_{6}-0.7836 a_{5} a_{7}+0.3512 a_{5} a_{8}-$ $4.128 a_{5} a_{9}+0.4528 a_{5} a_{10}+1.097 a_{5} a_{11}+0.1628 a_{5} a_{12}+$ $0.1927 a_{6} a_{7}-0.0233 a_{6} a_{8}+0.9535 a_{6} a_{9}-0.02632 a_{6} a_{10}-$ $0.1283 a_{6} a_{11}-0.1928 a_{6} a_{12}+0.1288 a_{7} a_{8}-2.389 a_{7} a_{9}+$ $0.2781 a_{7} a_{10}+0.7983 a_{7} a_{11}+0.1287 a_{7} a_{12}+0.8273 a_{8} a_{9}-$ $0.02315 a_{8} a_{10}-0.1045 a_{8} a_{11}-0.01245 a_{8} a_{12}+1.348 a_{9} a_{10}+$ $3.314 a_{9} a_{11}+0.3812 a_{9} a_{12}-0.2247 a_{10} a_{11}-0.04672 a_{10} a_{12}-$ $0.07624 a_{11} a_{12}$.

\section{Acknowledgement}

The work described in this paper is partially supported by the NSF of China (No. 61472356, 61210007), and the National Key Research and Development Program (2016YFC1101302) from the MIST of China.

\section{References}

[1] L. J. Gibson. Mechanical behavior of metallic foams. Annual Review of Materials Science, 30:191-227, 2000.

[2] R. M. Christensen. Mechanics of cellular and other low-density materials. International Journal of Solids and Structures, 37(1-2):93$104,2000$.

[3] T. J. Lu, Audrey Hess, and M. F. Ashby. Sound absorption in metallic foams. Journal of Applied Physics, 85(11):7528-7539, 1999.

[4] T. Pritz. Dynamic young's modulus and loss factor of plastic foams for impact sound isolation. Journal of Sound and Vibration, 178(3):315-322, 1994.

[5] I. Elnasri, S. Pattofatto, H. Zhao, H. Tsitsiris, F. Hild, and Y. Girard. Shock enhancement of cellular structures under impact loading: Part I Experiments. Journal of the Mechanics and Physics of Solids, 55(12):2652-2671, 2007.

[6] S. L. Lopatnikov, B. A. Gama, M. J. Haque, C. Krauthauser, J. W. Gillespie, M. Guden, and I. W. Hall. Dynamics of metal foam deformation during Taylor cylinder-Hopkinson bar impact experiment. Composite Structures, 61(1-2):61-71, 2003.

[7] I. S. Golovin and H. R. Sinning. Damping in some cellular metallic materials. Journal of Alloys and Compounds, 355(1-2):2-9, 2003.

[8] J. Banhart, J. Baumeister, and M. Weber. Damping properties of aluminium foams. Materials Science and Engineering A, 205(95):221228, 1996.

[9] E. W. Andrews and L. J. Gibson. The influence of cracks, notches and holes on the tensile strength of cellular solids. Acta Materialia, 49(49):2975-2979, 2001.

[10] N. A. Fleck and M. F. Ashby. Micro-architectured materials: past, present and future. Proceedings of the Royal Society A, 466(2121):2495-2516, 2010.

[11] M. F. Ashby and R. F. M. Medalist. The mechanical properties of cellular solids. Metallurgical and Materials Transactions A, 14(9):1755-1769, 1983.

[12] A. G. Evans, J. W. Hutchinson, N. A. Fleck, M. F. Ashby, and H. N. G. Wadley. The topological design of multifunctional cellular metals. Progress in Materials Science, 46(3-4):309-327, 2001.

[13] H. N. G. Wadley. Multifunctional periodic cellular metals. Philosophical Transactions of the Royal Society A Mathematical Physical and Engineering Sciences, 364(1838):31-68, 2006.

[14] A. G. Evans, J. W. Hutchinson, and M. F. Ashby. Multifunctionality of cellular metal systems. Progress in Materials Science, 43(3):171221, 1998.

[15] A. C. To, J. Tao, M. Kirca, and L. Schalk. Ligament and joint sizes govern softening in nanoporous aluminum. Applied Physics Letters, 98(5):051903-051903-3, 2011.

[16] A. Giri, J. Tao, L. Wang, M. Kirca, and A. C. To. Compressive behavior and deformation mechanism of nanoporous open-cell foam with ultrathin ligaments. Journal of Nanomechanics and Micromechanics, 4(2):A4013012, 2013.

[17] M. Wang and N. Pan. Predictions of effective physical properties of complex multiphase materials. Materials Science and Engineering: R: Reports, 63(1):1-30, 2008.

[18] S. M. Giusti, A. A. Novotny, E. A. D. S. Neto, and R. A. Feijóo. Sensitivity of the macroscopic elasticity tensor to topological microstructural changes. Journal of the Mechanics and Physics of Solids, 57(3):555-570, 2009.

[19] J. T. B. Overvelde, S. Shan, and K. Bertoldi. Compaction through buckling in 2D periodic, soft and porous structures: effect of pore shape. Advanced Materials, 24(17):2337-42, 2012.

[20] F. Göncü, S. Willshaw, J. Shim, J. Cusack, S. Luding, T. Mullin, and K. Bertoldi. Deformation induced pattern transformation in a soft granular crystal. Soft Matter, 7(6):2321, 2011.

[21] K. Bertoldi, P. M. Reis, S. Willshaw, and T. Mullin. Novel negative poisson's ratio behavior induced by an elastic instability. Advanced Materials, 22(3):361-6, 2010.

[22] S. Babaee, J. Shim, Weaver J. C., E. R. Chen, N. Patel, and K. Bertoldi. 3D soft metamaterials with negative poisson's ratio. Advanced Materials, 25(36):5044-5049, 2013.

[23] L. Wang and K. Bertoldi. Mechanically tunable phononic band gaps in three-dimensional periodic elastomeric structures. International Journal of Solids and Structures, 49(19-20):2881-2885, 2012. 
[24] F. Casadei and K. Bertoldi. Harnessing fluid-structure interactions to design self-regulating acoustic metamaterials. Journal of Applied Physics, 115(3):034907, 2014

[25] J. H. Panchal, S. R. Kalidindi, and D. L. McDowell. Key computational modeling issues in Integrated Computational Materials Engineering. Computer-Aided Design, 45(1):4-25, 2013.

[26] H. F. Al-Harbi, G. Landi, and S. R. Kalidindi. Multi-scale modeling of the elastic response of a structural component made from a composite material using the materials knowledge system. Modeling and Simulation in Materials Science and Engineering, 20:055001, 2012

[27] T. Fast, M. Knezevic, and S. R. Kalidindi. Application of microstructure sensitive design to structural components produced from hexagonal polycrystalline metals. Computational Materials Science, 43(2):374-383, 2008.

[28] H. Xu, Y. Li, Y. Chen, and J. Barbic. Interactive material design using model reduction. ACM Transactions on Graphics (TOG), 34(2):18, 2015

[29] H. Ghasemi, R. Rafiee, X. Zhuang, J. Muthu, and T. Rabczuk. Uncertainties propagation in metamodel-based probabilistic optimization of $\mathrm{CNT} /$ polymer composite structure using stochastic multi-scale modeling. Computational Materials Science, 85:295-305, 2014.

[30] H. Ghasemi, P. Kerfriden, S. P. A. Bordas, J. Muthu, G. Zi, and T. Rabczuk. Probabilistic multiconstraints optimization of cooling channels in ceramic matrix composites. Composites Part B: Engineering, 81:107-119, 2015.

[31] H. Ghasemi, R. Brighenti, X. Zhuang, J. Muthu, and T. Rabczuk. Optimal fiber content and distribution in fiber-reinforced solids using a reliability and NURBS based sequential optimization approach. Structural and Multidisciplinary Optimization, 51(1):99-112, 2015.

[32] M. A. Akgün, J. H. Garcelon, and R. T. Haftka. Fast exact linear and non-linear structural reanalysis and the Sherman-MorrisonWoodbury formulas. International Journal for Numerical Methods in Engineering, 50(7):1587-1606, 2001.

[33] D. T. Fullwood, S. R. Niezgoda, B. L. Adams, and S. R. Kalidindi. Microstructure sensitive design for performance optimization. Progress in Materials Science, 55(6):477-562, 2010.

[34] B. L. Adams, S. R. Kalidindi, and D. T. Fullwood. Microstructure sensitive design for performance optimization. ButterworthHeinemann, 2013.

[35] H. Xu, Y. Li, C. Brinson, and W. Chen. Descriptor-based methodology for designing heterogeneous microstructural materials system. In ASME 2013 International Design Engineering Technical Conferences and Computers and Information in Engineering Conference, pages V03AT03A049-V03AT03A049. American Society of Mechanical Engineers, 2013.

[36] Y. Liu, M. S. Greene, W. Chen, D. A. Dikin, and W. K. Liu. Computational microstructure characterization and reconstruction for stochastic multiscale material design. Computer-Aided Design, 45(1):65-76, 2013.

[37] H. Xu, D. A. Dikin, C. Burkhart, and W. Chen. Descriptor-based methodology for statistical characterization and 3D reconstruction of microstructural materials. Computational Materials Science, 85:206-216, 2014.

[38] O. Sigmund and K. Maute. Topology optimization approaches: a comparative review. Structural and Multidisciplinary Optimization, 48(6):1031-1055, 2013.

[39] J. Yan, G. Cheng, L. Liu, and S. Liu. Concurrent material and structural optimization of hollow plate with truss-like material. Structural and Multidisciplinary Optimization, 35(2):153-163, 2008.

[40] P. Zhang, J. Toman, Y. Yu, E. Biyikli, M. Kirca, M. Chmielus, and A. C. To. Efficient design-optimization of variable-density hexagonal cellular structure by additive manufacturing: Theory and Validation. Journal of Manufacturing Science and Engineering, 137(2):021004, 2015

[41] W. Wang, T. Y. Wang, Z. Yang, L. Liu, X. Tong, W. Tong, J. Deng, F. Chen, and X. Liu. Cost-effective printing of 3D objects with skinframe structures. ACM Transactions on Graphics (TOG), 32(6):1-10, 2013.
42] L. Lu, A. Sharf, H. Zhao, Y. Wei, Q. Fan, X. Chen, Y. Savoye, C. Tu, D. Cohen-Or, and B. Chen. Build-to-last: Strength to weight 3D printed objects. ACM Transactions on Graphics (TOG), 33(4):97, 2014.

[43] J. Panetta, Q. Zhou, L. Malomo, N. Pietroni, P. Cignoni, and D. Zorin. Elastic textures for additive fabrication. ACM Transactions on Graphics (TOG), 34(4):135, 2015.

[44] C. Schumacher, B. Bickel, J. Rys, S. Marschner, C. Daraio, and M. Gross. Microstructures to control elasticity in 3D printing. ACM Transactions on Graphics (TOG), 34(4), 2015.

[45] S. Li, J. Huang, F. de Goes, X. Jin, H. Bao, and M. Desbrun. Spacetime editing of elastic motion through material optimization and reduction. ACM Transactions on Graphics (TOG), 33:1-10, 2014.

[46] M. P. Bendsøe and N. Kikuchi. Generating optimal topologies in structural design using a homogenization method. Computer methods in applied mechanics and engineering, 71(2):197-224, 1988.

[47] L. J. Gibson and M. F. Ashby. Cellular solids: structure and properties. Cambridge university press, 1997.

[48] A. Mordecai. Nonlinear Programming: Analysis and Methods. Courier Corporation, 2003.

[49] K. Svanberg. The method of moving asymptotes-a new method for structural optimization. International Journal for Numerical Methods in Engineering, 24(2):359-373, 1987. 